\title{
Transceiver Design for MIMO Systems with Individual Transmit Power Constraints
}

\author{
Raja Muthalagu \\ Department of EEE, BITS, Pilani, Dubai Campus, Dubai, UAE- 345055.
}

\begin{tabular}{l} 
Article Info \\
\hline Article history: \\
Received: Aug 17, 2017 \\
Revised: Feb 21, 2018 \\
Accepted: Mar 13, 2018 \\
\hline Keyword: \\
MIMO \\
individual transmit power \\
constraint (ITPC) \\
channel state information \\
(CSI)
\end{tabular}

\begin{abstract}
This paper investigate the transceiver design for single-user multiple-input multipleoutput system (SU-MIMO). Joint transceiver design with an improper modulation is developed based on the minimum total mean-squared error (TMSE) criterion under two different cases. One is equal power allocation (EPA) and other is the power constraint that jointly meets both EPA and total transmit power constraint (TTPC) (i.e ITPC). Transceiver is designed based on the assumption that both the perfect and imperfect channel state information (CSI) is available at both the transmitter and receiver. The simulation results show the performance improvement of the proposed work over conventional work in terms of bit error rate (BER).
\end{abstract}

Copyright (c) 2018 Institute of Advanced Engineering and Science. All rights reserved.

\section{Corresponding Author:}

Raja Muthalagu

Department of EEE, BITS, Pilani, Dubai Campus

Dubai, UAE

+971-562249227

raja.sanjeeve@gmail.com

\section{INTRODUCTION}

\subsection{Background}

Multiple-input multiple-output (MIMO) systems are widely used to substantially increase the spectral efficiency of wireless channels. However, the benefits of multi-user MIMO highly depend on the type of channel state information (CSI) at both ends and on the level of accuracy of this information. Practical high data rates wireless systems can only have imperfect CSI at the receiver(CSIR), i.e., an estimate of the channel based on training sequence.

Multiple-input multiple-output (MIMO) systems are widely used to substantially increase the spectral efficiency of wireless. The spectral efficiency of the MIMO systems is increased linearly with the increase in the number of transmit and receive antennas. However, the efficiency of MIMO system highly rely on whether channel state information (CSI) at both ends or not. In practice, getting the perfect CSI is impractical because of the dynamic nature of the channel and the channel estimation errors. Thusly, it is important to outline a system sufficiently enough to imperfect CSIT and/or CSIR. An MIMO systems can be sub-divided into three fundamental classifications, spatial diversity $[1,2,3,4]$, spatial multiplexing $[5,6,7]$ and beamforming $[8,9,10]$.

In single-user multiple-input multiple-output(SU-MIMO) system, the diversity can be got through the utilization of space-time codes $[11,12]$. To accomplish full diversity, the transmit beamforming with receive combining was one of the least difficult methodologies. To enable spatial multiplexing in SU-MIMO systems, the appropriate transmit precoding design or joint precoder-decoder designs were proposed under a variety of system objectives and different CSI assumptions. Another beamforming method utilizing singular value decomposition (SVD) for closed loop SU-MIMO systems with a convolution encoder and modulation 
techniques, for example, $M$-quadrature amplitude modulation ( $M$-QAM) and $M$-phase shift keying ( $M$-PSK) over the Rayleigh fading have been proposed in our past works.

As far as spectral effectiveness, a SU-MIMO system ought to be intended to approach the capacity of the channel $[13,14,15]$. In the light of this perception, a frequency-selective MIMO channel can be managed by taking a multicarrier approach, which is a well-known capacity lossless the structure and permits us to treat every carrier a flat MIMO channel. A capacity- achieving design manages that the channel matrix at every carrier must be diagonalized, and afterward, a water-filling power distribution must be utilized on the spatial subchannels of all carriers. Note that this obliges CSI available at both the receiver and transmitter.

As design criteria, different performance measures are considered, for example, Weighted Minimum MSE [16, 17], TMSE [18], least BER [19]. From signal processing point of view, so as to minimize the information estimation error from the received signal, TMSE is a critical metric for transceiver design and has been embraced in SU-MIMO systems.A joint transceiver design for a SU-MIMO frameworks, utilizing an MSE paradigm is presented in [16]. A novel optimization method is proposed to solve the probabilistic MSE constrained multiuser multiple-input single-output (MU-MISO) transceiver design problem [20].

\subsection{The Problem}

All the schemes that are introduced in the above works is general and addressed a few optimization criteria like extreme data rate, least BER, and MMSE. The issue of designing an optimum linear transceiver for a SU-MIMO channel, possibly with delay spread, utilizing a weighted MMSE paradigm subject to a transmit power constraint is composed in [16]. These studies assume that the perfect CSI was available at the transmitter side. However, in practical communication systems, the propagation environment may be more challenging, and the receiver and transmitter can not have a perfect knowledge of the CSI. The imperfect CSI may emerge from an assortment of sources, for example, outdated channel estimates, error in channel estimation, quantization of the channel estimate in the feedback channel and so forth [21].

To obtain a robust communications system, the MIMO systems design with imperfect CSI is an important issue to investigate. The optimal precoding strategies in SU-MIMO systems were proposed under the assumption that imperfect CSI is available at the transmitter, and perfect CSI is available at the transmitter [22]. The robust joint precoder and decoder design to reduce the TMSE with imperfect CSI at both the transmitter and receiver of SU-MIMO systems were proposed in [23, 24, 25].

A novel precoding techniques to enhance the performance of the downlink in MU-MIMO system was studied with improper constellation [26]. Precoding designed in [26] is more appropriate for a MIMO system with improper signal constellation. MMSE and modified zero-forcing (ZF) precoder designs are demonstrated to accomplish an unrivaled performance than the routine linear and non-linear precoders. Both instances of imperfect and perfect CSI are considered, where the imperfect CSI case considers the correlation data and channel mean.

A joint precoder and decoder design under the minimum TMSE measure produced exceptional BER performance for, proper constellation techniques, e.g., $M$-PSK and $M$-QAM $[27,28]$. Then again, when applying the same outline to the improper constellation techniques, e.g., $M$-ASK and BPSK, the performance corrupts fundamentally. The minimum TMSE design for SU-MIMO system with improper modulation techniques was proposed in [26] and indicated to give a predominant performance in terms of BER than the traditional design in [27]. The optimum joint precoder and decoder designs for the SU-MIMO frameworks which utilize improper constellation strategies, either under the imperfect or perfect CSI was proposed in [29, 30, 31]. In both instances of imperfect and perfect CSI, a minimum TMSE measure is created and used to develop an iterative design technique for the optimum precoding and decoding matrices [29, 30, 31].

\subsection{The Proposed Solution}

In all of these designs only the TMSE measure is considered. The TMSE measure leads to wide power variations across the transmit antennas and poses severe constraint on the power amplifier design. However, to the best of our knowledge, no attention has been paid to either the ITPC or EPA based joint SU-MIMO transceiver design which employ improper modulation techniques, either under the perfect CSI or imperfect CSI assumption. To fill the gap, this paper shall address the problem of designing jointly optimum SU-MIMO transceiver under improper modulation that minimize the sum of symbol estimation error subject to EPA and 
ITPC(i.e jointly optimize the TTPC and EPA). It assumes the perfect and imperfect CSI with correlation information is available at both the transmitter and receiver. An iterative design procedure is developed to find the optimum precoding and decoding matrices.

The rest of the paper is organized as follows. The system model, power constraint and problem formulation are presented in Section 2. The proposed optimum joint precoder and decoder design for SU-MIMO under imperfect CSI is presented in Section 3. The simulation results for the proposed system is presented in Section 4. Finally conclusions are given in Section 5.

Notations: Throughout this paper, $(\cdot)^{T}$ denotes matrix transpose, upper (lower) case boldface letters are for matrices (vectors), $(\cdot)^{H}$ stands for matrix conjugate transpose, $E(\cdot)$ is expectation, $(\cdot)^{*}$ means matrix conjugate, $\|\cdot\|$ is Euclidian norm, $\mathbf{I}_{N}$ is an $N \times N$ identity matrix and $\operatorname{Tr}(\cdot)$ is the trace operation.

\section{SYSTEM MODEL, POWER CONSTRAINT AND PROBLEM FORMULATION}

\subsection{System Model}

A general SU-MIMO system model consist of $M_{\mathrm{T}}$ transmit and $M_{\mathrm{R}}$ receive antennas. The input bit streams are modulated by some improper modulation techniques to generate symbol streams. The symbol streams to be sent are denoted by a $B \times 1$ vector $\mathbf{s}=\left[S_{1}, \ldots, S_{B}\right]^{T}$, where $B$ is the number of data streams (i.e) $B=\operatorname{rank}(\mathbf{H}) \leq \min \left(M_{\mathrm{R}}, M_{\mathrm{T}}\right)$, where $\mathbf{H}$ is $M_{\mathrm{R}} \times M_{\mathrm{T}}$ channel matrix with its $(i, j)$ th element $h_{\mathrm{i}, \mathrm{j}}$ denoting the channel response from the $i$ th transmit antenna and $j$ th receive antenna. The modulated symbols are passed through the precoding matrix $\mathbf{U}$ of size $M_{\mathrm{T}} \times B$ to produce a $M_{\mathrm{T}} \times 1$ precoded vector $\mathbf{x}=\mathbf{U} \mathbf{s}$. The precoding matrix with the complex components adds redundancy to the modulated symbol to enhance the MIMO system performance. The precoded vector is passed through the MIMO channel through $N_{\mathrm{T}}$ antennas. The data symbols are assumed to be uncorrelated and have zero mean and unit energy, i.e., $E\left[\mathbf{s s}^{H}\right]=\mathbf{I}_{B}$. At the receiver end, received signal at receiving antennas are processed by the linear decoder matrix Vof size $B \times M_{\mathrm{R}}$.

For a MIMO channel without any delay-spread, the $M_{\mathrm{R}} \times 1$ received signal vector is defined as

$$
\mathbf{y}=\mathbf{H} \mathbf{x}+\mathbf{n}
$$

$\mathbf{y}$ is fed to the decoder $\mathbf{V}$. Then the resultant vector is:

$$
\hat{\mathbf{s}}=\mathbf{V H U s}+\mathbf{V n}
$$

where the $M_{\mathrm{R}} \times 1$ vector nrepresents spatially and temporally additive white Gaussian noise (AWGN) of zero mean and variance $\sigma_{n}^{2}$.

\subsection{Power Constraint}

The conventional joint precoder and decoder design are based on the following TTPC [29]:

$$
E\left[\|\mathbf{x}\|^{2}\right]=E\left[\|\mathbf{U s}\|^{2}\right]=\operatorname{Tr}\left(\mathbf{U} \mathbf{U}^{H}\right)=P .
$$

where $P$ is the total transmitted power from all the antennas at the transmitter. Most of the precoding or joint precoding and decoding design for the MIMO systems is studied with TTPC across all antennas. Here, we consider the more realistic ITPC. The $p$-norm concept is a multitasking algorithm, and the different power allocation can be obtained by changing the value of $p$. In linear algebra theory, the P-norm is given by [32, 33]

$$
\|x\|_{p}:=\left(\sum_{i=1}^{B}\left|x_{i}\right|^{p}\right)^{1 / p} \text { forp } \geq 1
$$

1. For $p=1,\|x\|_{1}:=\sum_{i=1}^{B}\left|x_{i}\right|^{1}$. This is 1-norm and it is simply the sum of the absolute value of $x_{i}$. So this referes to TTPC if $x_{i}$ denotes the power in each antenna.

2. For $p=\infty,\|x\|_{\infty}:=\max \left(x_{1}, \ldots, x_{M}\right)$. In linear algebra theory, this infinity norm is a special case of uniform norm, so this refers to equal power allocation (EPA). 
3. For $1<p<\infty$, the $p$-norm constraint is formulated as an optimization problem and can satisfy both the TTPC and EPA with an appropriate value for $p$, so this refers to individual transmit power constraint (ITPC).

\subsection{Problem Formulation}

The optimum joint precoder and decoder for SU-MIMO systems which employing a proper modulation techniques (e.g., $M$-PSK, $M$-ASK for which $E\left[\mathbf{s s}^{T}\right]=\mathbf{0}$ ) is derived by minimizing the TMSE under the TTPC specified by (??). The TMSE matrix is calculated as

$$
e=E\left[\|\hat{\mathbf{s}}-\mathbf{s}\|^{2}\right]=E\left[\|(\mathbf{V H U} \mathbf{s}+\mathbf{V n})-\mathbf{s}\|^{2}\right]
$$

This TMSE criterion expressed in (5) is optimum for the SU-MIMO systems with proper modulations. In any case, with improper modulation techniques (for which $E\left[\mathbf{s s}^{T}\right] \neq \mathbf{0}$ ) considered in this work, the TMSE criterion for SU-MIMO systems design expressed by (5) is not optimum. Since the traditional methodology expressed by (5) yields a complex-esteemed filter output. But, only the real part of this output is relevant for the decision in an MIMO system with improper constellations [30]. In this work, the MIMO design under TTPC in [31] is extended to both the EPA and ITPC.

By considering the improper constellations, the error vector is expressed as follows:

$$
\mathbf{e}=\hat{\mathbf{s}}-\mathbf{s}
$$

where $\hat{\mathbf{s}}=\Re(\mathbf{V H U s}+\mathbf{V n})$. Watch that the estimation of the received signal $\hat{\mathbf{s}}$ is changed from the conventional design expressed in (5). Thusly, the MSE criterion with respect to only the real part of the received signal with TTPC will result in a better design.

With the newly defined error vector, the TMSE can be computed as follows:

$$
\begin{aligned}
& E\left[\|\mathbf{e}\|^{2}\right]=E\left[\|\Re(\mathbf{V H U} \mathbf{s}+\mathbf{V n})-\mathbf{s}\|^{2}\right] \\
& =E\left[\|\left(\mathbf{V H U} \mathbf{\mathbf { s }}+\mathbf{V}^{*} \mathbf{H}^{*} \mathbf{U}^{*} \mathbf{s}^{*}\right) / 2+(\mathbf{V} \mathbf{n}\right. \\
& \left.\left.+\mathbf{V}^{*} \mathbf{n}^{*}\right) / 2-\mathbf{s} \|^{2}\right] \\
& =\operatorname{Tr}\left\{E \left\{\left[0.5\left(\mathbf{V H U} \mathbf{s}+\mathbf{V}^{*} \mathbf{H}^{*} \mathbf{U}^{*} \mathbf{s}^{*}\right)\right.\right.\right. \\
& \left.\quad+0.5\left(\mathbf{V n}+\mathbf{V}^{*} \mathbf{n}^{*}\right)-\mathbf{s}\right] \\
& \\
& {\left[0.5\left(\mathbf{s}^{H} \mathbf{U}^{H} \mathbf{H}^{H} \mathbf{V}^{H}+\mathbf{s}^{T} \mathbf{U}^{T} \mathbf{H}^{T} \mathbf{V}^{T}\right)\right.} \\
& \left.\left.\left.\quad+0.5\left(\mathbf{n}^{H} \mathbf{V}^{H}+\mathbf{n}^{T} \mathbf{V}^{T}\right)-\mathbf{s}^{H}\right]\right\}\right\}
\end{aligned}
$$

we consider the following assumptions on the statistics of the data, noise and channel (i.e. $E[\mathbf{n}]=$ $E\left[\mathbf{n n}^{T}\right]=E\left[\mathbf{n}^{*} \mathbf{n}^{H}\right]=\mathbf{0}, E\left[\mathbf{n n}^{H}\right]=\sigma_{n}^{2} \mathbf{I}_{\mathrm{N}_{T}}$ and $\left.E\left[\mathbf{s s}^{H}\right]=E\left[\mathbf{s s}^{T}\right]=\mathbf{I}_{B}\right)$. By using those assumption and after some manipulation (8) can be simplified to

$$
\begin{aligned}
& E\left[\|\mathbf{e}\|^{2}\right]= \\
& \operatorname{Tr}\left\{0 . 2 5 \left(\mathbf{V H} \mathbf{U} \mathbf{U}^{H} \mathbf{H}^{H} \mathbf{V}^{H}\right.\right. \\
&+\mathbf{V H} \mathbf{U} \mathbf{U}^{T} \mathbf{H}^{T} \mathbf{V}^{T} \\
&\left.+\mathbf{V}^{*} \mathbf{H}^{*} \mathbf{U}^{*} \mathbf{U}^{H} \mathbf{H}^{H} \mathbf{V}^{H}\right) \\
&\left.+\mathbf{V}^{*} \mathbf{H}^{*} \mathbf{U}^{*} \mathbf{U}^{T} \mathbf{H}^{T} \mathbf{V}^{T}\right) \\
&-0.5\left(\mathbf{V} \mathbf{H} \mathbf{U}+\mathbf{V}^{*} \mathbf{H}^{*} \mathbf{U}^{*}+\mathbf{U}^{H} \mathbf{H}^{H} \mathbf{V}^{H}\right. \\
&\left.+\mathbf{U}^{T} \mathbf{H}^{T} \mathbf{V}^{T}\right)+\mathbf{I}_{B} \\
&\left.+0.25 \sigma_{n}^{2}\left(\mathbf{V} \mathbf{V}^{H}+\mathbf{V}^{*} \mathbf{V}^{T}\right)\right\}
\end{aligned}
$$

The goal is to find an optimum $\mathbf{U}$ and $\mathbf{V}$ which minimize $E\left[\|\mathbf{e}\|^{2}\right]$ subject to the TTPC, total transmit power $\left(\operatorname{Tr}\left(\mathbf{U} \mathbf{U}^{H}\right)\right.$ and the transmit power constraint that jointly optimize the TTPC and EPA (i.e ITPC). 
Mathematically, it is defined as

$$
\begin{gathered}
\min _{\mathbf{U}, \mathbf{V}} E\left[\|\Re(\mathbf{V H U} \mathbf{\mathbf { s }}+\mathbf{V n})-\mathbf{s}\|^{2}\right] \\
\text { s.t. } \quad\left(\operatorname{Tr}\left(\mathbf{U} \mathbf{U}^{H}\right)^{p}\right)^{1 / p} \leq P_{\mathrm{T}} .
\end{gathered}
$$

where $P_{\mathrm{T}}$ is a constant. If $p=1$, then the $P_{\mathrm{T}}$ will be equal to TTPC $(\beta), p=\infty$ corresponds to an EPA $(\alpha)$ where $\alpha=\beta / B$. And for $p$ in the interval $1<p<\infty, P_{\mathrm{T}}=\infty$, $p$-norm condition is sufficient to meet both the EPA and a TTPC with upper bound $\beta$. The $\alpha$ can be chosen between the interval of $[\beta / B, \beta]$. Here, we form the Lagrangian to find the optimum solution to optimization problem expressed in (10)

$$
\begin{aligned}
\psi= & E\left[\|\Re(\mathbf{V H U} \mathbf{s}+\mathbf{V n})-\mathbf{s}\|^{2}\right] \\
& +\chi\left(\left(\operatorname{Tr}\left(\mathbf{U} \mathbf{U}^{H}\right)^{p}\right)^{1 / p}-P_{\mathrm{T}}\right)
\end{aligned}
$$

where $\chi$ is the Lagrange multiplier. The optimum values for precoder and decoder matrix are found by following the same procedure as in [31].

\section{PROPOSED MATHOS: OPTIMUM JOINT PRECODER AND DECODER DESIGN FOR SU- MIMO WITH IMPERFECT CSI}

This section proposes a design of the optimum linear precoder and decoder for SU-MIMO system employing improper modulations based on the power constraint that jointly optimize TTPC and EPA. Here it consider the imperfect CSI along with the transmit and receive correlation is available at both the end. We use the channel model in our previous work [31], that is

$$
\mathbf{H}=\mathbf{R}_{\mathrm{R}}^{1 / 2} \mathbf{H}_{\mathrm{w}} \mathbf{R}_{\mathrm{T}}^{1 / 2}
$$

where $\mathbf{H}_{\mathrm{w}}$ is a spatially white matrix whose entries are independent and identically distributed (i.i.d.) $\mathcal{N}_{c}(0,1)$. The matrices $\mathbf{R}_{R}$ and $\mathbf{R}_{T}$ represent the normalized receive and transmit correlations, respectively. Both transmit and receive correlations are assumed to be full-rank and known to both the receiver and the transmitter. The orthogonal training method [29] is performed to estimate the channel error. It can be described as follows

$$
\mathbf{H}=\hat{\mathbf{H}}+\mathbf{E}
$$

where $\hat{\mathbf{H}}=\mathbf{R}_{\mathrm{R}}^{1 / 2} \hat{\mathbf{H}}_{\mathrm{w}} \mathbf{R}_{\mathrm{T}}^{1 / 2}$ is the estimated overall channel matrix, $\hat{\mathbf{H}}_{\mathrm{w}}$ is the MMSE estimation of $\mathbf{H}_{\mathrm{w}}, \mathbf{E}=\mathbf{R}_{\mathrm{e}, \mathrm{R}}^{1 / 2} \mathbf{E}_{\mathrm{w}} \mathbf{R}_{\mathrm{T}}^{1 / 2}$ is the channel estimation error matrix, $\mathbf{R}_{\mathrm{e}, \mathrm{R}}^{1 / 2}=\left[\mathbf{I}_{M_{\mathrm{R}}}+\sigma_{\mathrm{ce}}^{2} \mathbf{R}_{\mathrm{R}}^{-1}\right]^{-1}$ is the effect of the receive correlation on the channel estimation error, $\sigma_{\text {ce }}^{2}$ is the quality of the channel estimate and the entries of $\mathbf{E}_{\mathrm{w}}$ are i.i.d. $\mathcal{N}_{c}\left(0, \sigma_{\text {ce }}^{2}\right)$.

By modeling the true channel as in (13) under the MMSE channel estimation, the TMSE function for joint transceiver design can be evaluated for improper modulation as follows:

$$
\begin{aligned}
& E\left[\|\mathbf{e}\|^{2}\right]=E\left[\|\hat{\mathbf{s}}-\mathbf{s}\|^{2}\right] \\
& \quad=E\left[\|\Re(\mathbf{V}(\hat{\mathbf{H}}+\mathbf{E}) \mathbf{U} \mathbf{s}+\mathbf{V n})-\mathbf{s}\|^{2}\right] \\
&=E\left[\|\left(\mathbf{V}(\hat{\mathbf{H}}+\mathbf{E}) \mathbf{U} \mathbf{s}+\mathbf{V}^{*}(\hat{\mathbf{H}}+\mathbf{E})^{*} \mathbf{U}^{*} \mathbf{s}^{*}\right) / 2\right. \\
&\left.\quad+\left(\mathbf{V} \mathbf{n}+\mathbf{V}^{*} \mathbf{n}^{*}\right) / 2-\mathbf{s} \|^{2}\right] \\
& \quad=\operatorname{Tr}\left\{E \left\{\left[0.5\left(\mathbf{V}(\hat{\mathbf{H}}+\mathbf{E}) \mathbf{U s}+\mathbf{V}^{*}(\hat{\mathbf{H}}+\mathbf{E})^{*} \mathbf{U}^{*} \mathbf{s}^{*}\right)+\right.\right.\right. \\
&\left.\quad{ }^{\prime} 0.5\left(\mathbf{V} \mathbf{n}+\mathbf{V}^{*} \mathbf{n}^{*}\right)-\mathbf{s}\right] \\
& {\left[0.5\left(\mathbf{s}^{H} \mathbf{U}^{H}(\hat{\mathbf{H}}+\mathbf{E})^{H} \mathbf{V}^{H}+\mathbf{s}^{T} \mathbf{U}^{T}(\hat{\mathbf{H}}+\mathbf{E})^{T} \mathbf{V}^{T}\right)\right.} \\
&\left.\left.\left.+0.5\left(\mathbf{n}^{H} \mathbf{V}^{H}+\mathbf{n}^{T} \mathbf{V}^{T}\right)-\mathbf{s}^{H}\right]\right\}\right\}
\end{aligned}
$$


Substituting $\mathbf{E}=\mathbf{R}_{\mathrm{e}, \mathrm{R}}^{1 / 2} \mathbf{E}_{\mathrm{w}} \mathbf{R}_{\mathrm{T}}^{1 / 2}$ in (14) and after taking expectation with respect to $\mathbf{s}, \mathbf{E}_{\mathrm{w}}$, and $\mathbf{n}$, (14) becomes $^{1}$ :

$$
\begin{aligned}
& E\left[\|\mathbf{e}\|^{2}\right]= \\
& \operatorname{Tr}\left\{0.25 \mathbf{V} \hat{\mathbf{H}} \mathbf{U} \mathbf{U}^{H} \hat{\mathbf{H}}^{H} \mathbf{V}^{H}+0.25 \mathbf{V} \hat{\mathbf{H}} \mathbf{U} \mathbf{U}^{T} \hat{\mathbf{H}}^{T} \mathbf{V}^{T}\right. \\
&-0.5 \mathbf{V} \hat{\mathbf{H}} \mathbf{}+0.25 \mathbf{V} \mathbf{R}_{\mathrm{e}, \mathrm{R}} \mathbf{V}^{H} \operatorname{Tr}\left(\mathbf{R}_{\mathrm{T}} \mathbf{U} \mathbf{U}^{H}\right) \sigma_{\mathrm{ce}}^{2} \\
&+0.25 \mathbf{V} \mathbf{V}^{H} \sigma_{n}^{2}+0.25 \mathbf{V}^{*} \hat{\mathbf{H}}^{*} \mathbf{U}^{*} \mathbf{U}^{H} \hat{\mathbf{H}}^{H} \mathbf{V}^{H} \\
&+0.25 \mathbf{V}^{*} \hat{\mathbf{H}}^{*} \mathbf{U}^{*} \mathbf{U}^{T} \hat{\mathbf{H}}^{T} \mathbf{V}^{T}-0.5 \mathbf{V}^{*} \hat{\mathbf{H}}^{*} \mathbf{U}^{*} \\
&+0.25 \mathbf{V}^{*} \mathbf{R}_{\mathrm{e}, \mathrm{R}}^{*} \mathbf{V}^{T}\left\{\operatorname{Tr}\left(\mathbf{R}_{\mathrm{T}} \mathbf{U}^{H}\right)\right\}^{*} \sigma_{\mathrm{ce}}^{2}+0.25 \mathbf{V}^{*} \mathbf{V}^{T} \sigma_{n}^{2} \\
&\left.-0.5 \mathbf{U}^{H} \hat{\mathbf{H}}^{H} \mathbf{V}^{H}-0.5 \mathbf{U}^{T} \hat{\mathbf{H}}^{T} \mathbf{V}^{T}+\mathbf{I}_{B}\right\}
\end{aligned}
$$

By substituting (15) in (11) and taking the derivatives of $\psi$ with respect to $\mathbf{V}$ and $\mathbf{U}$, it can be shown that the associated Karush-Kuhn-Tucker (KKT) conditions can be obtained and given in the following.:

First, the value of $\frac{\partial \psi}{\partial \mathbf{V}}$ can be found by using the cyclic property of the trace function. Setting $\frac{\partial \psi}{\partial \mathbf{V}}=0$ and taking the complex conjugates of both sides gives

$$
\begin{array}{r}
\mathbf{V}\left(\hat{\mathbf{H}} \mathbf{U} \mathbf{U}^{H} \hat{\mathbf{H}}^{H}+\mathbf{R}_{\mathrm{e}, \mathrm{R}} \sigma_{\mathrm{ce}}^{2} \operatorname{Tr}\left(\mathbf{R}_{\mathrm{T}} \mathbf{U U}^{H}\right)\right) \\
+\mathbf{V}^{*} \hat{\mathbf{H}}^{*} \mathbf{U}^{*} \mathbf{U}^{H} \hat{\mathbf{H}}^{H}+\sigma_{n}^{2} \mathbf{V}=2 \mathbf{U}^{H} \hat{\mathbf{H}}^{H}
\end{array}
$$

Similarly, setting $\frac{\partial \psi}{\partial \mathbf{U}}=0$. Again, taking the complex conjugates of both sides has gives

$$
\begin{array}{r}
\left(\hat{\mathbf{H}}^{H} \mathbf{V}^{H} \mathbf{V} \hat{\mathbf{H}}+\mathbf{R}_{\mathrm{T}} \sigma_{\mathrm{ce}}^{2} \operatorname{Tr}\left(\mathbf{R}_{\mathrm{e}, \mathrm{R}} \mathbf{V}^{H} \mathbf{V}\right)\right) \mathbf{U} \\
+\hat{\mathbf{H}}^{H} \mathbf{V}^{H} \mathbf{V}^{*} \hat{\mathbf{H}}^{*} \mathbf{U}^{*}+2 \chi\left(\operatorname{Tr}\left(\mathbf{U} \mathbf{U}^{H}\right)^{p}\right)^{1 / p} \\
=2 \hat{\mathbf{H}}^{H} \mathbf{V}^{H}
\end{array}
$$

Next, by post-multiplying both sides of (16) by $\mathbf{V}^{H}$ one obtains

$$
\begin{array}{r}
\left(\mathbf{V}\left(\hat{\mathbf{H}} \mathbf{U} \mathbf{U}^{H} \hat{\mathbf{H}}^{H}+\mathbf{R}_{\mathrm{e}, \mathrm{R}} \sigma_{\mathrm{ce}}^{2} \operatorname{Tr}\left(\mathbf{R}_{\mathrm{T}} \mathbf{U}^{H}\right)\right)\right. \\
\left.+\mathbf{V}^{*} \hat{\mathbf{H}}^{*} \mathbf{U}^{*} \mathbf{U}^{H} \hat{\mathbf{H}}^{H}+\sigma_{n}^{2} \mathbf{V}\right) \mathbf{V}^{H}=2 \mathbf{V}^{H} \mathbf{U}^{H} \hat{\mathbf{H}}^{H}
\end{array}
$$

Likewise, pre-multiplying both sides of (17) by $\mathbf{U}^{H}$ produces

$$
\begin{array}{r}
\left(\left(\hat{\mathbf{H}}^{H} \mathbf{V}^{H} \mathbf{V} \hat{\mathbf{H}}+\mathbf{R}_{\mathrm{T}} \sigma_{\mathrm{ce}}^{2} \operatorname{Tr}\left(\mathbf{R}_{\mathrm{e}, \mathrm{R}} \mathbf{V}^{H} \mathbf{V}\right)\right) \mathbf{U}\right. \\
\left.+\hat{\mathbf{H}}^{H} \mathbf{V}^{H} \mathbf{V}^{*} \hat{\mathbf{H}}^{*} \mathbf{U}^{*}+2 \chi\left(\operatorname{Tr}\left(\mathbf{U} \mathbf{U}^{H}\right)^{p}\right)^{1 / p}\right) \mathbf{U}^{H} \\
=2 \hat{\mathbf{H}}^{H} \mathbf{V}^{H} \mathbf{U}^{H}
\end{array}
$$

It then follows from (18) and (19) that:

$$
\begin{array}{r}
\left(\mathbf{V}\left(\hat{\mathbf{H}} \mathbf{U} \mathbf{U}^{H} \hat{\mathbf{H}}^{H}+\mathbf{R}_{\mathrm{e}, \mathrm{R}} \sigma_{\mathrm{ce}}^{2} \operatorname{Tr}\left(\mathbf{R}_{\mathrm{T}} \mathbf{U U}^{H}\right)\right)\right. \\
\left.+\mathbf{V}^{*} \hat{\mathbf{H}}^{*} \mathbf{U}^{*} \mathbf{U}^{H} \hat{\mathbf{H}}^{H}+\sigma_{n}^{2} \mathbf{V}\right) \mathbf{V}^{H} \\
=\left(\left(\hat{\mathbf{H}}^{H} \mathbf{V}^{H} \mathbf{V} \hat{\mathbf{H}}+\mathbf{R}_{\mathrm{T}} \sigma_{\mathrm{ce}}^{2} \operatorname{Tr}\left(\mathbf{R}_{\mathrm{e}, \mathrm{R}} \mathbf{V}^{H} \mathbf{V}\right)\right) \mathbf{U}\right. \\
\left.+\hat{\mathbf{H}}^{H} \mathbf{V}^{H} \mathbf{V}^{*} \hat{\mathbf{H}}^{*} \mathbf{U}^{*}+2 \chi\left(\operatorname{Tr}\left(\mathbf{U} \mathbf{U}^{H}\right)^{p}\right)^{1 / p}\right) \mathbf{U}^{H}
\end{array}
$$

\footnotetext{
${ }^{1}$ In performing the expectation, the following results are used: $E\left[\mathbf{E}_{\mathrm{w}}\right]=E\left[\mathbf{E}_{\mathrm{w}}^{H}\right]=\mathbf{0}, E\left[\mathbf{E}_{\mathrm{w}} \mathbf{A} \mathbf{E}_{\mathrm{w}}^{H}\right]=\sigma_{\mathrm{ce}}^{2} \operatorname{tr}(\mathbf{A}) \mathbf{I}_{N}$ and $E\left[\mathbf{E}_{\mathrm{w}} \mathbf{A} \mathbf{E}_{\mathrm{w}}^{T}\right]=\mathbf{0}$.
} 
Then, by taking the traces of both sides of (20) one has:

$$
\chi=\frac{\sigma_{n}^{2}}{2 P_{\mathrm{T}}} \operatorname{Tr}\left(\mathbf{V} \mathbf{V}^{H}\right)
$$

As in [31], the optimum solution for precoding and decoding matrix are obtained by using the explicit relationship between (16), (17) and (21). An iterative algorithm is used to find the solutions. The optimum decoding matrix is

$$
\begin{gathered}
{\left[\begin{array}{ll}
\mathbf{V}_{\mathrm{Re}} & \mathbf{V}_{\mathrm{Im}}
\end{array}\right]=\left[\begin{array}{cc}
\mathbf{C}_{\mathrm{Re}} & \mathbf{C}_{\mathrm{Im}}
\end{array}\right]} \\
{\left[\begin{array}{cc}
A B & \mathbf{A}_{\mathrm{Im}}+\mathbf{B}_{\mathrm{Im}} \\
\mathbf{B}_{\mathrm{Im}}-\mathbf{A}_{\mathrm{Im}} & A C
\end{array}\right]}
\end{gathered}
$$

where $A B=\mathbf{A}_{\mathrm{Re}}+\mathbf{B}_{\mathrm{Re}}+\sigma_{n}^{2} \mathbf{I}_{N_{\mathrm{R}}}, A C=\mathbf{A}_{\mathrm{Re}}+\mathbf{B}_{\mathrm{Re}}-\sigma_{n}^{2} \mathbf{I}_{N_{\mathrm{R}}} \mathbf{V}=\mathbf{V}_{\mathrm{Re}}+j \mathbf{V}_{\mathrm{Im}}, \mathbf{H} \mathbf{U U}^{H} \mathbf{H}^{H}=$ $\mathbf{A}_{\mathrm{Re}}+j \mathbf{A}_{\mathrm{Im}}, \mathbf{H}^{*} \mathbf{U}^{*} \mathbf{U}^{H} \mathbf{H}^{H}=\mathbf{B}_{\mathrm{Re}}+j \mathbf{B}_{\mathrm{Im}}$ and $2 \mathbf{F}^{H} \mathbf{H}^{H}=\mathbf{C}_{\mathrm{Re}}+\mathbf{C}_{\mathrm{Im}}$.

Similarly, the optimum precoding matrix is

$$
\begin{array}{r}
{\left[\begin{array}{c}
\mathbf{U}_{\mathrm{Re}} \\
\mathbf{U}_{\mathrm{Im}}
\end{array}\right]=\left[\begin{array}{cc}
A D & \mathbf{Q}_{\mathrm{Im}}-\mathbf{P}_{\mathrm{Im}} \\
\mathbf{P}_{\mathrm{Im}}+\mathbf{Q}_{\mathrm{Im}} & A E
\end{array}\right]^{-1}} \\
{\left[\begin{array}{c}
\mathbf{R}_{\mathrm{Re}} \\
\mathbf{R}_{\mathrm{Im}}
\end{array}\right]}
\end{array}
$$

where $A D=\mathbf{P}_{\mathrm{Re}}+\mathbf{Q}_{\mathrm{Re}}+2 \mu \mathbf{I}_{N_{\mathrm{T}}}, A E=\mathbf{P}_{\mathrm{Re}}+\mathbf{Q}_{\mathrm{Re}}-2 \chi \mathbf{I}_{N_{\mathrm{T}}} \mathbf{U}=\mathbf{U}_{\mathrm{Re}}+j \mathbf{U}_{\mathrm{Im}}, \mathbf{H}^{H} \mathbf{V}^{H} \mathbf{G H}=$ $\mathbf{P}_{\mathrm{Re}}+j \mathbf{P}_{\mathrm{Im}}, \mathbf{H}^{H} \mathbf{V}^{H} \mathbf{V}^{*} \mathbf{H}^{*}=\mathbf{Q}_{\mathrm{Re}}+j \mathbf{Q}_{\mathrm{Im}}$ and $2 \mathbf{H}^{H} \mathbf{V}^{H}=\mathbf{R}_{\mathrm{Re}}+\mathbf{R}_{\mathrm{Im}}$

Based on the above expressions, the optimum precoder and decoder can be solved by an iteration procedure as outlined in following algorithm:

1. Initialize $\mathbf{U}$, and $\mathbf{U}$ by setting the $B \times B$ upper sub-matrix of $\mathbf{U}$ a scaled identity matrix (which satisfies the power constraint with equality), while all the other remaining entries of $\mathbf{U}$ are zero.

2. Find the value of $\mathbf{V}$ using (22).

3. Find the value of $\chi$ using (21).

4. Find U using (23).

5. If $\left(\operatorname{Tr}\left(\mathbf{U} \mathbf{U}^{H}\right)^{p}\right)^{1 / p} \leq P_{\mathrm{T}}$ for $1<p<\infty$, scale $\mathbf{U}$ such that $\operatorname{Tr}\left\{\mathbf{U} \mathbf{U}^{H}\right\}=P_{\mathrm{T}}$, else go to the next step.

6. If $\left(\operatorname{Tr}\left(\left(\mathbf{U}^{i}-\mathbf{U}^{i-1}\right)\left(\mathbf{U}^{i}-\mathbf{U}^{i-1}\right)^{H}\right)^{p}\right)^{1 / p}<10^{-4}$, then terminate, else go to Step 2 .

\section{RESULTS AND DISCUSSION}

This section presents the performance of the proposed transceiver design for SU-MIMO system employing improper modulation, EPA and ITPC. The Matlab simulation has been used for modelling the proposed SU-MIMO system and channel. The performance of the proposed system over the imperfect CSI is measured in terms of BER. The BPSK, 4-ASK and QPSK are applied to modulate the data. To illustrate the performance improvement of the proposed system, the BER performance of the SU-MIMO system with improper modulation under TTPC [31] is compared with the proposed SU-MIMO system with improper modulation under both ITPC and EPA. The simulation results are averaged over at least 10,000 channel realizations. In all the simulation results reported in this section, the number of parallel date streams are set as $B=4$ and the number of transmit and receiver antennas are fixed as $M_{\mathrm{T}}=M_{\mathrm{R}}=4$. The transmit correlation matric is defined as $\mathbf{R}_{\mathrm{T}}(i, j)=\rho_{\mathrm{T}}^{|i-j|}$ for $i, j=1,2, \ldots, M_{\mathrm{T}}$, where receive correlation metric is defined as $\mathbf{R}_{\mathrm{R}}(i, j)=\rho_{\mathrm{R}}^{|i-j|}$ for $i, j=1,2, \ldots, M_{\mathrm{R}}$. 


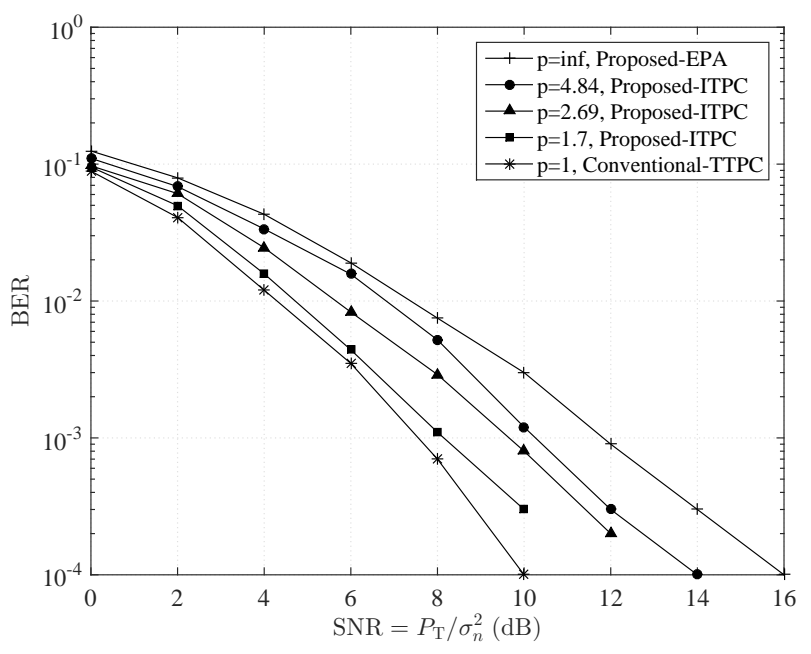

Figure 1. Performance comparison of the conventional transceiver and proposed transceiver for BPSK modulations and perfect CSI. $M_{\mathrm{T}}=M_{\mathrm{R}}=4, B=4, \sigma_{\mathrm{ce}}^{2}=0, \rho_{\mathrm{T}}=\rho_{\mathrm{R}}=0.0$.

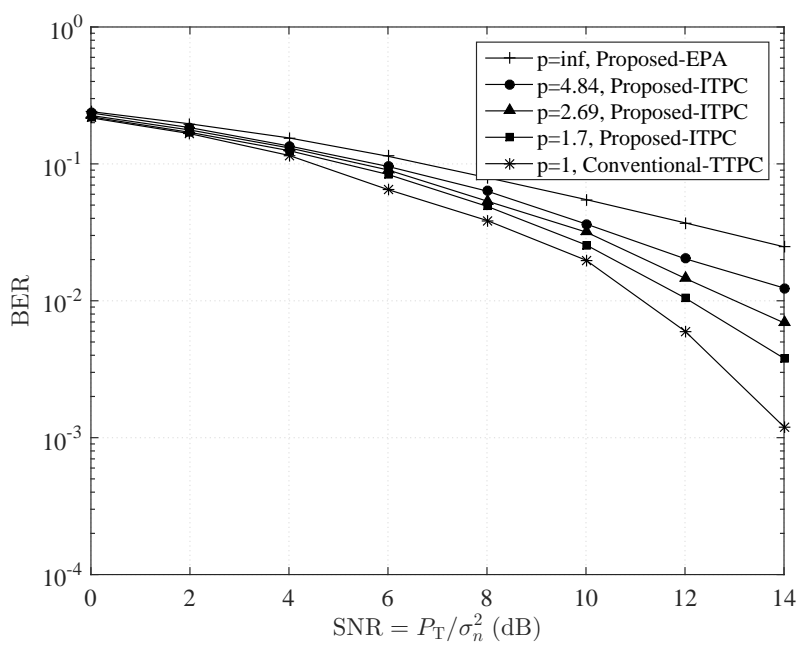

Figure 2. Performance comparison of the conventional transceiver and proposed transceiver for 4-ASK modulations and perfect CSI. $M_{\mathrm{T}}=M_{\mathrm{R}}=4, B=4, \sigma_{\text {ce }}^{2}=0, \rho_{\mathrm{T}}=\rho_{\mathrm{R}}=0.0$.

The SNR for all the simulation results in this paper is defined as $\mathrm{SNR}=\frac{P_{\mathrm{T}}}{\sigma_{n}^{2}}$ and the training phase $\mathrm{SNR}$ is defined as $\mathrm{SNR}_{\mathrm{tr}}=\frac{P_{\mathrm{tr}}}{\sigma_{n}^{2}}=26.016 \mathrm{~dB}$. The number of iteration required to converge the optimum value precoder decoder may vary between 6 to 9 iterations and it is mainly based on the SNR and channel condition. For the value of $p=1$ corresponds to the conventional TTPC, where the $p=\infty$ corresponds to the proposed EPA. For case $p$ between 0 and $\infty$ corresponds to the practical solution that satisfies ITPC. For the case of ITPC, three different values for $\alpha$ and $\beta$ are considered based on the $p$ value. Note that, with $p=\{1.7,2.69,4.84\}$, one has $\alpha=\{5.2,2.8,1.5\}$ and $\beta=\{9.8,6.7,4.5\}$.

First, Fig. 1 shows the performance comparisons of the conventional TTPC based linear SU-MIMO transceiver design for improper modulation in [31] with that of the proposed ITPC and EPA based linear SUMIMO transceiver design for improper modulation. The BPSK modulation is applied to modulate the data, and it assumes the perfect CSI is available at both the transmitter and receiver. The main purpose of this simulation to show the performance in terms of BER of the proposed ITPC and EPA based linear SU-MIMO transceiver design for improper modulation. As can be seen from the Fig. 1, the proposed EPA based SUMIMO system system leads to a SNR performance degradation of about $4 \mathrm{~dB}$ for BER $10^{-3}$ when compared to the conventional TTPC base SU-MIMO system. For $\mathrm{p}=1.7,2.69$ and 4.84 of the proposed ITPC based 


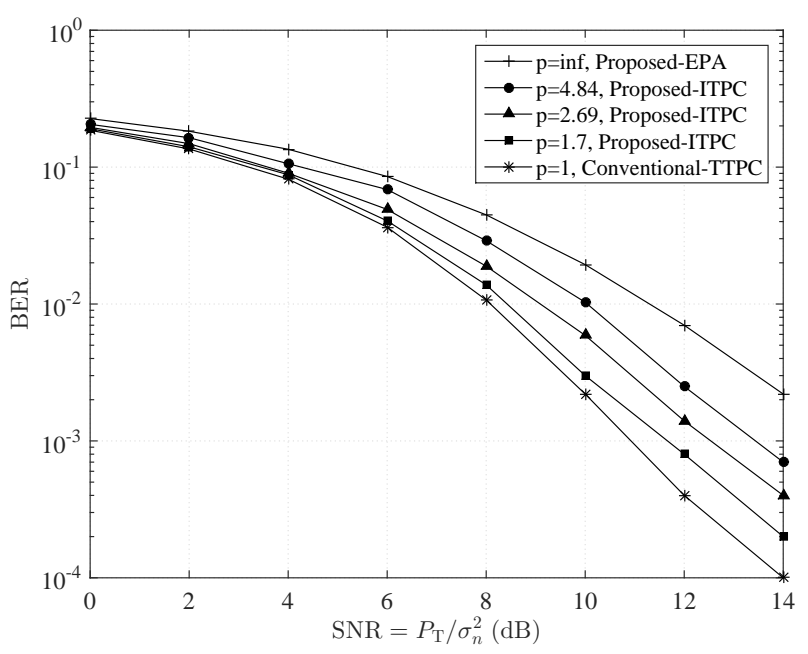

Figure 3. Performance comparison of the conventional transceiver and proposed transceiver for OQPSK modulations and perfect CSI. $M_{\mathrm{T}}=M_{\mathrm{R}}=4, B=4, \sigma_{\mathrm{ce}}^{2}=0, \rho_{\mathrm{T}}=\rho_{\mathrm{R}}=0.0$.

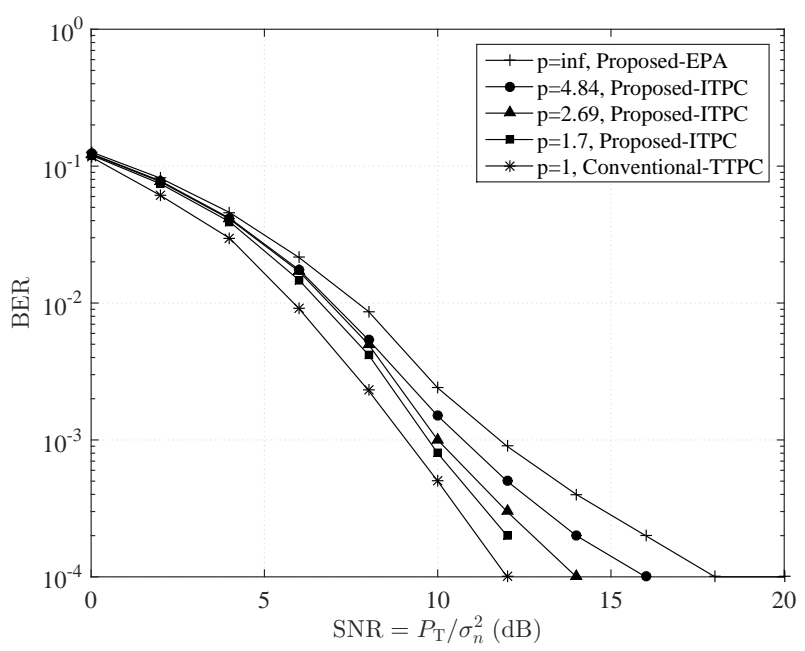

Figure 4. Performance comparison of the conventional transceiver and proposed transceiver for for BPSK and imperfect CSI. $M_{\mathrm{T}}=M_{\mathrm{R}}=4, B=3$ or $B=4, \sigma_{\mathrm{ce}}^{2}=0.015, \rho_{\mathrm{T}}=\rho_{\mathrm{R}}=0.5$.

SU-MIMO system, $1 \mathrm{~dB}, 2 \mathrm{~dB}$ and $3 \mathrm{~dB}$ SNR degradation is observed, correspondingly when compared to the conventional TTPC based linear SU-MIMO system.

This comparison shows the conventional TTPC based SU-MIMO system achieves a little superior performance than the proposed methods. However, in practice, EPA and ITPC based SU-MIMO system is more suitable as the power at each transmit antenna is limited individually by the linearity of the power amplifier. In that way all the four types of proposed methods are prepared to design a SU-MIMO transceiver rather than conventional TTPC based SU-MIMO system. Note also that, since perfect CSI is available at both the transmitter and receiver ends, the performance curves improve exponentially with SNR and there is no error floor in all performance curves. Fig. 2 and Fig. 3 also shows the similar type of performance comparisons as in Fig. 1 but for the case of 4-ASK and OQPSK, correspondingly under perfect CSI. Again, the performance degradation of our proposed design over the conventional design is clearly observed from Fig. 2 and Fig. 3.

Fig. 4, Fig. 5 and Fig. 6 shows performance comparisons of the conventional TTPC based linear SUMIMO transceiver design for improper modulation in [31] with that of the proposed ITPC and EPA based linear SU-MIMO transceiver design for BPSK, 4-ASK and OQPSK, respectively but for the case of imperfect CSI. As mentioned before the MIMO system design takes into account the one-dimensional property of improper 


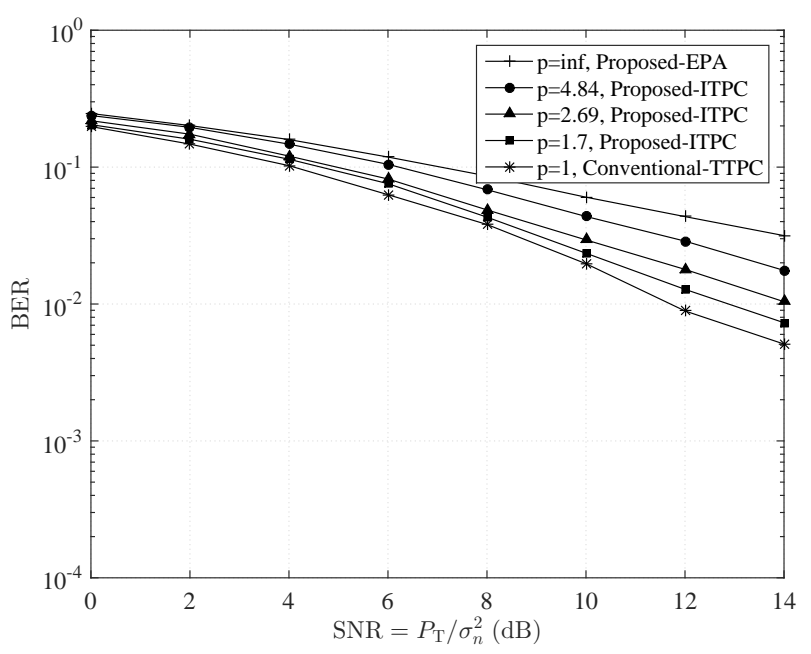

Figure 5. Performance comparison of the conventional transceiver and proposed transceiver for for 4-ASK and imperfect CSI. $M_{\mathrm{T}}=M_{\mathrm{R}}=4, B=3$ or $B=4, \sigma_{\mathrm{ce}}^{2}=0.015, \rho_{\mathrm{T}}=\rho_{\mathrm{R}}=0.5$.

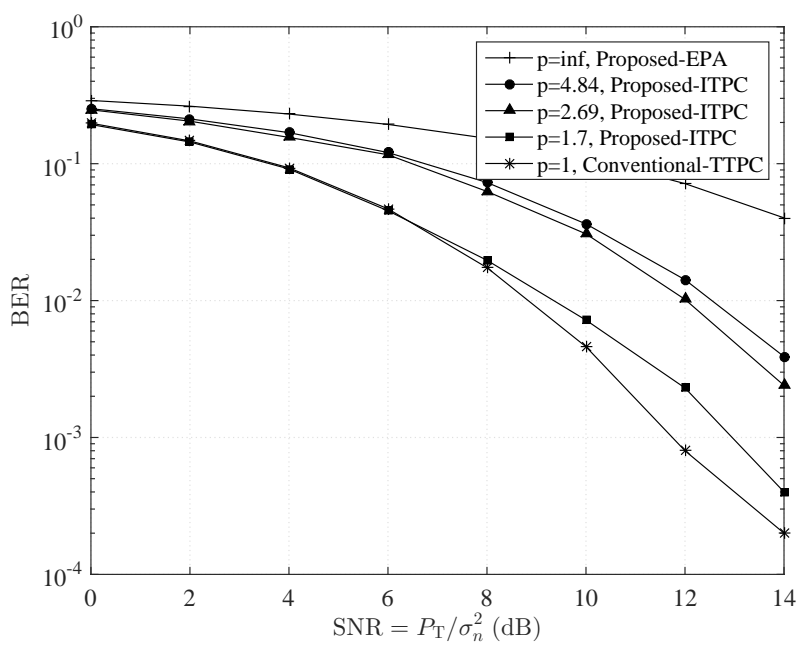

Figure 6. Performance comparison of the conventional transceiver and proposed transceiver for for OQPSK and imperfect CSI. $M_{\mathrm{T}}=M_{\mathrm{R}}=4, B=3$ or $B=4, \sigma_{\text {ce }}^{2}=0.015, \rho_{\mathrm{T}}=\rho_{\mathrm{R}}=0.5$.

modulations. As can be seen from the figure, the proposed joint linear transceiver leads to a little performance degradation, especially for EPA based SU-MIMO system with BPSK modulation (an SNR degradation of about $3 \mathrm{~dB}$ is observed for BER of $10^{-3}$ ). For the case of imperfect CSI following values are assumed for correlation $\rho_{\mathrm{T}}=\rho_{\mathrm{R}}=0.5$. Note that, with $\mathrm{SNR}_{\mathrm{tr}}=\frac{P_{\mathrm{tr}}}{\sigma_{n}^{2}}=26.016 \mathrm{~dB}$ and $\rho_{\mathrm{T}}=0.5$, one has $\sigma_{\text {ce }}^{2}=0.015$.

We also presented the performance comparison for BPSK, 4-ASK and OQPSK for proposed ITPC based SU-MIMO under both the perfect and imperfect CSI which are illustrated in Fig. 7. For value of $p=4.84$, one has $\alpha=1,2$ and $\beta=4.5$. Results of Fig. 7 show the effect of CSI on proposed design in terms of the BER. It is observed the fact that the proposed design for BPSK, 4-ASK and OQPSK has much better BER performance in perfect CSI, and the channel estimation errors cause a large performance degradation on the BER.

Fig. 8 examines the effect of channel correlations on the proposed system BER performance under imperfect CSI. For this figure, OQPSK modulation is employed with the number of data streams $B=4$. Various sets of transmit/receive correlations considered are $\left\{\rho_{\mathrm{T}}=0.9, \rho_{\mathrm{R}}=0.9\right\} ;\left\{\rho_{\mathrm{T}}=0.9, \rho_{\mathrm{R}}=0.5\right\}$; $\left\{\rho_{\mathrm{T}}=0.5, \rho_{\mathrm{T}}=0.9\right\}$; and $\left\{\rho_{\mathrm{T}}=0.5, \rho_{\mathrm{T}}=0.5\right\}$. With $p=1.7$, one has $\alpha=5.2$ and $\beta=9.8$. In general, Fig. 8 shows that higher values of the transmit and receive correlations cause bigger performance losses. 


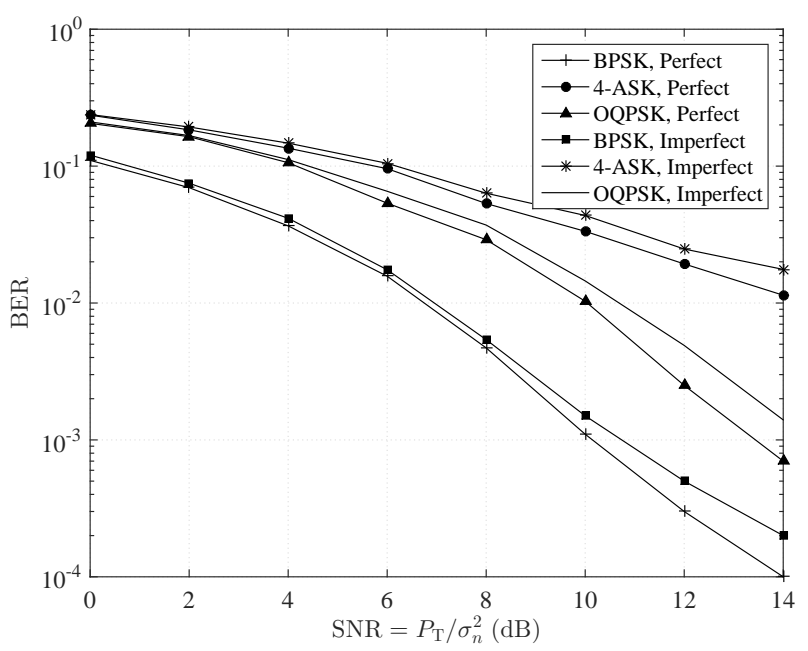

Figure 7. Performance of the proposed transceiver design with BPSK, 4-ASK and OQPSK under both the perfect and imperfect CSI. $M_{\mathrm{T}}=M_{\mathrm{R}}=4, B=4$. The values of $\sigma_{\text {ce }}^{2}$ are $0,0.015$ for $\rho_{\mathrm{T}}=0$ and $\rho_{\mathrm{T}}=0.5$, respectively.

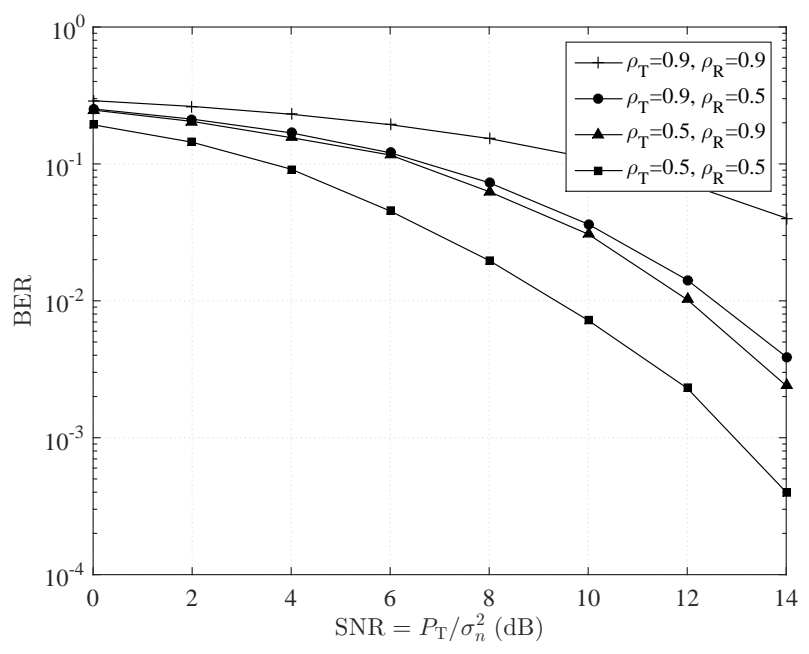

Figure 8. Effect of transmit and receive correlations on the performance of the proposed transceiver design with OQPSK. $M_{\mathrm{T}}=M_{\mathrm{R}}=4, B=4$. The values of $\sigma_{\text {ce }}^{2}$ are $0.015,0.0739$ for $\rho_{\mathrm{T}}=0.5$ and $\rho_{\mathrm{T}}=0.9$, respectively.

\section{CONCLUSION}

The proposed work in this paper addressed the designs of transceiver with improper constellations for SU-MIMO systems under both the perfect and imperfect CSI. The TMSE of the revived signal is formulated as nonconvex optimization problem under two different cases. One is equal power allocation (EPA) and other is the power constraint that jointly meets both EPA and total transmit power constraint (TTPC) (i.e ITPC). An iterative algorithm is used to find the optimum value for precoder and decoder in both the EPA and ITPC based design. Performance improvement of the proposed methods over conventional design in terms of the system's BER was thoroughly demonstrated by simulation results.

\section{REFERENCES}

[1] M. Kang and M. S. Alouini, "Performance analysis of MIMO MRC systems over Rician fading channels," Proc. of IEEE Vehic. Tech. Conf., pp. 869-873, 2002. 
[2] T. K. Y. Lo, “Maximum ratio transmission,”IEEE Trans. Commun, vol. 47, pp. 1458-1461, Oct. 1999.

[3] P. A. Dighe, R. K. Mallik and S. S. Jamuar, "Analysis of transmit-receive diversity in Rayleigh fading," IEEE Trans. Commun., vol. 51, pp. 694-703, Apr. 2003.

[4] S. N. Raut and R. M. Jalnekar, "Performance Enhancement in SU and MU MIMO-OFDM Technique for Wireless Communication: A Review," International Journal of Electrical and Computer Engineering (IJECE), vol. 7 (5), pp. 2459-2467, Oct. 2017.

[5] G. J. Foschini,"Layered space-time architecture for wireless communication in a fading environment when using multi-element antennas," Bell Labs Technical Journal, vol. 1, pp. 41-59, July. 1996.

[6] Adeeb Salh, Lukman Audah, Nor Shahida M. Shah and Shipun A. Hamzah,"Maximizing Energy Efficiency for Consumption Circuit Power in Downlink Massive MIMO Wireless Networks," International Journal of Electrical and Computer Engineering (IJECE), vol. 7 (6), pp. 2977-2985, Dec. 2017.

[7] L. Zhao, L. W. Mo, Y. Ma and Z. Wang , "Diversity and multiplexing trade-off in general fading channels," IEEE Trans. Inform. Theory, vol. 53, pp. 1549-1557, Apr. 2007.

[8] M. Raja and P. Muthuchidambaranathan,"BER Performance of SVD-based Transmit Beamforming with Various Modulation Techniques," Proc. of 5th International Conference on Industrial and Information Systems (ICIIS-2010), pp. 155-160, Jul. 2010.

[9] M. Raja and P. Muthuchidambaranathan, "SVD-Based Transmit Beamforming for Various Modulations with Convolution Encoding," ICTACT Journal on Communication Technology, vol. 2 pp. 393-399, Sep. 2011.

[10] M. Raja and P. Muthuchidambaranathan,"Performance Analysis of Closed-Loop MIMO system," International Journal of Computer Applications, vol. 4 pp. 14-19, Aug. 2011.

[11] S. M. Alamouti, "A simple transmit diversity technique for wireless communications," IEEE J. Select. Areas Commun., vol. 16, pp. 1451-1458, Oct. 1998.

[12] V. Tarokh, N. Seshadri and A. R. Calderbank, "Space-time codes for high data rate wireless communication: performance criterion and code construction," IEEE Trans. Information Theory, vol. 44, pp. 744-765, Mar. 1998.

[13] T. M. Cover and and J. A. Thomas, "Elements of Information Theory," Wiley, New York, 1991.

[14] Adeeb Salh, Lukman Audah, Nor Shahida M Shah and Shipun A Hamzah, "Adaptive Antenna Selection and Power Allocation in Downlink Massive MIMO Systems," International Journal of Electrical and Computer Engineering (IJECE), vol. 7 (6), pp.3521-3528, Dec. 2017.

[15] G. G. Raleigh and J. M. Cioffi“Spatio-temporal coding for wireless communication,” IEEE Trans. Commun., vol. 46, pp. 357-366, May. 1998.

[16] H. Sampath, P. Stoica, A. Paulraj, "Generalized linear precoder and decoder design for MIMO channels using the weighted MMSE criterion,'IEEE Trans. Communications, vol. 49, pp. 2198-2206, Dec. 2001.

[17] Saif Khan Mohammed, Erik G Larsson, "Improving the Performance of the Zero-Forcing Multiuser MISO Downlink Precoder Through User Grouping”, IEEE Transactions on Wireless Communications, 15(2), 811-826, 2016.

[18] A. Scaglione, P. Stoica, S. Barbarossa,G. B. Giannakis, H. Sampath "Optimal designs for space-time linear precoders and decoders,"IEEE Trans. Signal Processing, vol. 50, pp. 1051-1064, May. 2002.

[19] D. P. Palomar, "A Unified Framework for Communications through MIMO Channels," Ph.D. dissertation, Universitat Polit'ecnica de Catalunya (UPC), Barcelona, Spain, 2003.

[20] He, X, Wu, YC, "Tight Probabilistic MSE Constrained Multiuser MISO Transceiver Design under Channel Uncertainty,” Proc. IEEE Int. Conf. on Communication, pp. ‘4745-4750, 2015.

[21] Ramoni Adeogun, "Capacity and Error Rate Analysis of MIMO Satellite Communication Systems in Fading Scenarios," International Journal of Electrical and Computer Engineering (IJECE), vol. 4 (4), pp.614 - 622, Aug. 2014.

[22] H. Sampat and A. Paulraj, "Linear precoding for space-time coded systems with known fading correlations,'IEEE Commun. Lett., vol. 6, pp. 239-241, Jun. 2002.

[23] J. Zhang and Y. Wu, S. Zhou and J. Wang, "Joint linear transmitter and receiver design for the downlink of multiuser MIMO systems," IEEE Comm. Lett., vol. 9, pp. 991-993, Nov. 2005.

[24] X. Zhang and and D. P. Palomar, "Robust Design of Linear MIMO Transceivers Under Channel Uncertainty," Proc. IEEE Int. Conf. on Acoustics, Speech, and Signal Process., pp. IV77-IV80, May. 2006.

[25] Joonwoo Shin "Efficient transceiver design for large-scale antenna systems," EURASIP Journal on Wireless Communications and Networking, Springer, vol. 77, pp. 1251-1263, 2015. 
[26] Pei Xiao and M. Sellathurai, "Improved Linear Transmit Processing for Single-User and Multi-User MIMO Communications Systems," IEEE Trans. Signal Process., vol. 58, pp. 1768-1779, Mar. 2010.

[27] Minhua Ding and S. D. Blostein "MIMO Minimum Total MSE Transceiver Design With Imperfect CSI at Both Ends," IEEE Trans. Signal Process., vol. 57, pp. 1141-1150, Mar. 2009.

[28] Aki Hakkarainen, Janis Werner, Kapil R. Dandekar, Mikko Valkama "Precoded massive MU-MIMO uplink transmission under transceiver I/Q imbalance," IEEE Globecom Workshops , vol. 57, pp. 320 - 326 , Dec. 2014.

[29] K. Sanka , M. Raja and P. Muthuchidambaranathan "Improved minimum total MSE transceiver design with imperfect CSI at both ends of a MIMO link," IEEE International Conference on Electronics Computer Technology (ICECT), pp. 23-27, Apr. 2011.

[30] M. Raja, K. Sanka and P. Muthuchidambaranathan "Minimum Total MSE based Transceiver Design for Single-user MIMO System," Proc. of 17th IEEE Asia-Pacific Conference on Communications (APCC2011), pp. 720-725, Oct. 2011.

[31] M. Raja, P. Muthuchidambaranathan and Ha Nguyen "Transceiver Design for MIMO Systems with Improper Modulations," Wireless Personal Communications., Springer,vol. 68, pp. 265-280, Jan. 2013.

[32] Vu Mai "MIMO Capacity with Per-Antenna Power Constraint," Global Telecommunications Conference (GLOBECOM 2011), Proc. IEEE Global Telecommunications Conference (GLOBECOM ), pp. 1-5, Dec. 2011.

[33] A. Merline and S. J. Thiruvengadam "Design of Optimal Linear Precoder and Decoder for MIMO Channels with Per Antenna Power Constraint and Imperfect CSI," Wireless Personal Communications., Springer,vol. 75, pp. 1251-1263, Mar. 2014. 